\title{
Optimal Power Flow over Tree Networks
}

\author{
Subhonmesh Bose, Dennice F. Gayme, Steven Low, K. Mani Chandy
}

\begin{abstract}
The optimal power flow (OPF) problem is critical to power system operation but it is generally non-convex and therefore hard to solve. Recently, a sufficient condition has been found under which OPF has zero duality gap, which means that its solution can be computed efficiently by solving the convex dual problem. In this paper we simplify this sufficient condition through a reformulation of the problem and prove that the condition is always satisfied for a tree network provided we allow over-satisfaction of load. The proof, cast as a complex semi-definite program, makes use of the fact that if the underlying graph of an $n \times n$ Hermitian positive semi-definite matrix is a tree, then the matrix has rank at least $n-1$.
\end{abstract}

\section{Motivation}

Optimal operation of a power grid has been extensively studied since the pioneering work of Carpentier [1] in 1962. The general optimal power flow (OPF) problem seeks to minimize some cost function, such as power loss, generation cost and/or user utilities, subject to capacity and network constraints on the voltages, powers (real and reactive) and the loads [2]-[4]. The general OPF problem is non-convex and NP hard. Given the practical importance of the problem there has been a lot of research into efficient solution algorithms, and historically the most common solution techniques have relied on linear programming techniques [5], [6]. Researchers have also proposed a number of relaxations to make the OPF problem more tractable. The simplest of which is the DC Power Flow problem, which is widely used because it is a linear program and thus easy to solve. However this approximation makes a number of assumptions that are not always valid in a real power circuit. A number of studies have sought to characterize the instances where the DC approximation is acceptable, e.g. [7], [8] and the references therein. A detailed overview of some other common instances of OPF along with various solution strategies are provided in the survey articles [9]-[12].

Recently there has been some effort toward convexifying the full AC problem. Jabr provided a conic quadratic model of radial distribution systems [13] and meshed networks [14] and demonstrated an efficient solution method to these problems using an interior point method for convex conic quadratic programming. The implementation of this method on a distribution system containing various reactive power components such as tap changers and shunt capacitors was also studied [15]. The trigonometric angle constraints in

S. Bose is with Department of Electrical Engineering and D. F. Gayme, S. Low and K. M. Chandy are with Department of Computational and Mathematical Sciences at California Institute of Technology, Pasadena, CA 91125

\{bosese, dennicelcds., slowe, maniecs.\}caltech.edu

This work was supported by NSF through NetSE grant CNS 0911041, Southern California Edison, Cisco, and the Okawa Foundation. these works make the results difficult to generalize. For radial networks the method in [14] also requires the additional step of traversing the tree to recover the angles after the optimization problem is solved. In [16], [17], Baran and $\mathrm{Wu}$ introduced a new model for a radial network and an efficient computational method that makes use of the loopfree nature of such a network. Farivar et al. [18] builds on the model in [16], [17] and studies a second order cone relaxation to determine the optimal control strategy for the multi-timescale problem of simultaneous optimal inverter and shunt capacitor control and conjectures that the relaxation is exact. In all of these works the relaxations seem to perform well but no guarantees are provided as to the ability to recover the solution of the original problem nor is there a characterization of the worst-case distance from their solution to that of the original problem.

Bai et al. show in [19], [20] that under certain conditions the OPF problem can be cast as a semi-definite program. This idea was further refined and extensively analyzed by Lavaei et al. in [21], [22] who proved that these conditions always hold for resistive power networks and provided strong evidence that the method works for most practical circuits. In [22] the OPF is shown to be equivalent to a semidefinite program with a rank-constraint through transforming the voltage and power constraints, which are quadratic in nature, into linear matrix inequalities. The use of semidefinite relaxations for quadratically constrained quadratic programs has long been of interest in the literature [23] [25]. The technique is described in detail in [26], [27] and a number of applications have been studied, see e.g., [28], [29].

Instead of solving the OPF problem directly, [22] proposes to solve the Lagrangian dual problem [26], [30], which is an SDP. The dual of the SDP is a convex rank relaxation of the OPF problem, and since both the SDP and the rank relaxation are convex, strong duality holds between them. They prove a sufficient condition under which the rank relaxation is exact. This implies that the duality gap between OPF and its Lagrangian dual (SDP) is also zero, and hence an optimal primal solution to OPF can be obtained from an optimal solution of its Lagrangian dual. Even though many IEEE benchmark systems have been shown to satisfy the sufficient condition, a complete understanding as to why it holds for so many practical circuits remains elusive. In this paper we take a closer look at this condition in a simplified setting where the underlying graph of the power system is a tree (radial) network.

Radial networks are common in distribution circuits [31], [32]. As in [22], we construct the Lagrangian dual (which 
we denote by the problem $D P$ ) of the OPF problem using standard techniques, e.g. [26], [27], [30], and show that OPF turns out to be equivalent to the dual of $D P$ (the so-called $D D P$ ) with an additional (non-convex) rank constraint. We prove a sufficient condition (similar to [22]) that guarantees that solutions of $D D P$ satisfy the rank constraint and therefore closes the duality gap [27]. Our key result proves that the condition is always satisfied for a tree network provided the load is over-satisfactied. The proof, cast as a complex semidefinite program, makes use of the fact that if the underlying graphs of a certain $n \times n$ Hermitian matrix induced by the admittance matrix is a connected tree, then the matrix has rank at least $n-1$.

The paper is organized as follows. Section II formulates our OPF problem. Section III describes the solution approach and proves that the sufficient condition for zero duality gap is always satisfied if the underlying graph of the matrix is a connected tree (Section III-B) or if over-satisfaction of load is allowed (Section III-C). These results carry over when storage devices are integrated with the system as explained in Section III-D. Finally we conclude with some future directions of inquiry.

\section{PROBLEM FORMULATION}

Consider a distribution circuit modeled as a radial network with $n$ nodes (buses) and define $[n]:=\{1,2, \ldots, n\}$ as the set of nodes. There is an edge between nodes $i$ and $j$ if the corresponding buses $i$ and $j$ are connected. We denote the admittance-to-ground at bus $i$ by $y_{i i}$, and the admittance of the line by $y_{i j}=g_{i j}-j b_{i j}$ if buses $i$ and $j$ are connected. We assume both $g_{i j}>0$ and $b_{i j}>0$, i.e., the lines are resistive and inductive. The graph $\mathcal{G}(Y)$ of $Y$ is defined with $n$ vertices with an undirected edge between vertices $i \neq j$ if $Y_{i j} \neq 0$. (Note: The diagonal entries of $Y$ do not play a role in $\mathcal{G}(Y)$.) Using this notation we can state the key assumption of the paper,

Assumption 1: The graph $\mathcal{G}(Y)$ is a tree with $n-1$ edges. We define the quantities in our model as follows.

- $Y$ : the $n \times n$ admittance matrix defined as

$$
Y_{i j}= \begin{cases}y_{i i}+\sum_{j \sim i} y_{i j}, & \text { if } i=j \\ -y_{i j}, & \text { if } i \neq j \text { and } i \sim j \\ 0 & \text { otherwise, }\end{cases}
$$

where $i \sim j$ indicates that bus $i$ is connected to bus $j$. Note that $Y$ is symmetric but not necessarily Hermitian.

- $V$ and $I$ : The $n$-dimensional vectors of complex voltages and currents where $V_{k}, I_{k}$ respectively denote the voltage and the injection current at bus $k$. They are related by Kirchhoff's law i.e., $I=Y V$. The square of the voltage magnitude at bus $k$ is bounded as:

$$
\underline{W}_{k} \leq\left|V_{k}\right|^{2} \leq \bar{W}_{k} .
$$

- $S=P+\mathbf{i} Q$ : The vector of (complex) apparent power $S$, real power $P$, reactive power $Q$, respectively. They are related to the voltage and current at bus $k$ through the relation $S_{k}=P_{k}+\mathbf{i} Q_{k}=V_{k} I_{k}^{*}, k \in[n]$.
- $P_{k}^{D}$ and $Q_{k}^{D}$ : The real and reactive power demands at bus $k$. They are assumed to be fixed and given.

- $P_{k}^{G}$ and $Q_{k}^{G}$. The real and reactive power generation at bus $k$. They are decision variables and constrained to be within certain ranges depending on the generation capacity at each bus:

$$
\underline{P}_{k}^{G} \leq P_{k}^{G} \leq \bar{P}_{k}^{G} \text { and } \underline{Q}_{k}^{G} \leq Q_{k}^{G} \leq \bar{Q}_{k}^{G} .
$$

At each bus $k$ power must be balanced such that $P_{k}^{G}=$ $P_{k}^{D}+P_{k}$ and $Q_{k}^{G}=Q_{k}^{D}+Q_{k}$. Let

$$
\begin{array}{ll}
\underline{P}_{k}:=\underline{P}_{k}^{G}-P_{k}^{D}, & \bar{P}_{k}:=\bar{P}_{k}^{G}-P_{k}^{D} \\
\underline{Q}_{k}:=\underline{Q}_{k}^{G}-Q_{k}^{D}, & \bar{Q}_{k}:=\bar{Q}_{k}^{G}-Q_{k}^{D} .
\end{array}
$$

Then the power injections must satisfy

$$
\underline{P}_{k} \leq P_{k} \leq \bar{P}_{k}, \quad \underline{Q}_{k} \leq Q_{k} \leq \bar{Q}_{k} .
$$

Optimal operation may correspond to minimizing the power loss over the network, the total generation cost, or the average voltage levels while keeping them within a certain band. For distribution circuits, studies have shown that voltage reduction can produce significant energy savings [33]. We choose our objective function to be $\|V\|^{2}=\sum_{k}\left|V_{k}\right|^{2}$ but the results presented in this paper would work for any quadratic function of the form $V^{*} M V$ where $M$ is diagonal or $\mathcal{G}(M)$ is a tree. We neglect line limits for our analysis.

Finally, we relate the power injections to bus voltages. Let $e_{1}, e_{2}, \ldots, e_{n}$ be the standard basis vectors in $\mathbb{C}^{n}$, i.e., $e_{k}$ is the column vector with ' 1 ' in its $k^{\text {th }}$ position and ' 0 ' in the other $n-1$ positions. Let $J_{k}=e_{k} e_{k}^{*}$. Let $Y_{k}=e_{k} e_{k}^{*} Y$. Then

$$
\begin{aligned}
S_{k} & =e_{k}^{*} V I^{*} e_{k}=e_{k}^{*} V V^{*} Y^{*} e_{k}=\operatorname{tr}\left(V V^{*}\left(Y^{*} e_{k} e_{k}^{*}\right)\right) \\
& =\left(V^{*} Y_{k}^{*} V\right) \\
& =(V^{*} \underbrace{\left(\frac{Y_{k}^{*}+Y_{k}}{2}\right)}_{=\Phi_{k}} V)+\mathbf{i}(V^{*} \underbrace{\left(\frac{Y_{k}^{*}-Y_{k}}{2 j}\right)}_{=\Psi_{k}} V) .
\end{aligned}
$$

Since $\Phi_{k}$ and $\Psi_{k}$ are Hermitian matrices, the two quantities $V^{*} \Phi_{k} V$ and $V^{*} \Psi_{k} V$ are real numbers. Thus,

$$
\begin{aligned}
P_{k} & =V^{*} \Phi_{k} V=\operatorname{tr}\left(\Phi_{k} V V^{*}\right) \\
Q_{k} & =V^{*} \Psi_{k} V=\operatorname{tr}\left(\Psi_{k} V V^{*}\right) .
\end{aligned}
$$

Define the OPF problem as follows:

Primal Problem $(P)$ :

$$
\begin{array}{cl}
\underset{V}{\operatorname{minimize}} & V^{*} V \\
\text { subject to: } & \\
& \underline{P}_{k} \leq V^{*} \Phi_{k} V \leq \bar{P}_{k}, \quad k \in[n] \\
& \underline{Q}_{k} \leq V^{*} \Psi_{k} V \leq \bar{Q}_{k}, \quad k \in[n] \\
& \underline{W}_{k} \leq V^{*} J_{k} V \leq \bar{W}_{k}, \quad k \in[n],
\end{array}
$$

where (1), (2) and (3) are the constraints on real powers, reactive powers and voltages respectively. 


\section{CONDITIONS FOR ZERO DUALITY GAP}

The primal problem $P$ is a non-convex quadratically constrained quadratic program. The matrices involved are Hermitian but indefinite in general. This means that $P$ is hard to solve for large problem instances. To circumvent this difficulty, we follow the approach in [22] and take the following steps in the rest of this paper:

1) Construct the dual problem $D P$ of $P$. The dual problem is convex and therefore can be solved efficiently. In order to obtain a primal optimal solution to $P$ from a dual optimal solution the duality gap must be zero. Directly determining the duality gap between $P$ and $D P$ is hard.

2) Construct the dual problem $D D P$ of $D P$. Strong duality holds between $D P$ and $D D P$ if Slater's condition holds since both are convex problems.

3) Observe that problem $P$ is equivalent to $D D P$ with a rank constraint, i.e., $D D P$ is a convex relaxation of $P$. Therefore if any solution of $D D P$ satisfies the rank constraint, then it is also primal optimal.

4) Compute the optimal value of the convex problem $D P$, which by 2 , equals the optimal value of $D D P$. When the rank constraint from 3 holds the solution of $D D P$ is equivalent to the solution of $P$, i.e., the duality gap between $P$ and $D P$ is zero. In other words, we obtain an optimal solution of $P$ by solving $D P$; see description after Theorem 3.1.

In [22] a sufficient condition is proved for general networks that guarantees that a solution of problem $D D P$ indeed satisfies the rank constraint in 3 above and hence the duality gap between $P$ and $D P$ is zero. In this paper, we invoke Assumption 1 from Section II and study the sufficient condition in [22] for a radial (tree) network.

\section{A. Condition for general network}

Let $\bar{\lambda}_{k}, \underline{\lambda}_{k}$ be the Lagrange multipliers for the real power constraints in problem $P$ for the upper and lower inequalities. Similarly define $\bar{\mu}_{k}, \underline{\mu}_{k}$ for the reactive power constraints and $\bar{\gamma}_{k}, \underline{\gamma}_{k}$ for the voltage constraints. Define

$$
\lambda_{k}=\bar{\lambda}_{k}-\underline{\lambda}_{k}, \quad \mu_{k}=\bar{\mu}_{k}-\underline{\mu}_{k}, \quad \gamma_{k}=\bar{\gamma}_{k}-\underline{\gamma}_{k} .
$$

The dual of $P$ and its own dual are the following problems [27].

\section{Dual of $P(D P)$ :}

$$
\begin{array}{cc}
\underset{\bar{\lambda}, \underline{\lambda}, \bar{\mu}, \underline{\mu}, \bar{\gamma}, \underline{\gamma}}{\operatorname{maximize}} & \sum_{k}\left\{\underline{\lambda}_{k} \underline{P}_{k}-\bar{\lambda}_{k} \bar{P}_{k}+\underline{\mu}_{k} \underline{Q}_{k}-\bar{\mu}_{k} \bar{Q}_{k}\right. \\
& \left.+\underline{\gamma}_{k} \underline{W}_{k}-\bar{\gamma}_{k} \bar{W}_{k}\right\} \\
\text { subject to } & I+\sum_{k}\left(\lambda_{k} \Phi_{k}+\mu_{k} \Psi_{k}+\gamma_{k} J_{k}\right) \succeq 0 \\
& \bar{\lambda}_{k} \geq 0, \underline{\lambda}_{k} \geq 0 \text { for } k \in[n] \\
& \bar{\mu}_{k} \geq 0, \underline{\mu}_{k} \geq 0 \text { for } k \in[n] \\
& \bar{\gamma}_{k} \geq 0, \underline{\gamma}_{k} \geq 0 \text { for } k \in[n] .
\end{array}
$$

Dual of the dual problem $(D D P)$ :

$$
\begin{aligned}
& \underset{W}{\operatorname{minimize}} \operatorname{tr}(W) \\
& \text { subject to } \underline{P}_{k} \leq \operatorname{tr}\left(\Phi_{k} W\right) \leq \bar{P}_{k}, \quad k \in[n] \\
& \underline{Q}_{k} \leq \operatorname{tr}\left(\Psi_{k} W\right) \leq \bar{Q}_{k}, \quad k \in[n] \\
& \underline{W}_{k} \leq \operatorname{tr}\left(J_{k} W\right) \leq \bar{W}_{k}, \quad k \in[n] \\
& W \succeq 0 \text {, }
\end{aligned}
$$

where $W=V V^{*}$. Using the identity $\operatorname{tr}\left(V^{*} B V\right)=$ $\operatorname{tr}\left(B V V^{*}\right)=\operatorname{tr} B W$ for any matrix $B$, it is apparent that the primal problem $P$ is equivalent to $D D P$ with the additional constraint that rank $W=1$. Hence, as mentioned earlier, $D D P$ is a convex relaxation of $P$ and any rank-1 optimal $W_{*}$ for $D D P$ defines a unique optimal $V_{*}$ for $P$. In summary, provided Slater's condition is satisfied, we have

$$
\text { optimal value of } \begin{aligned}
P & \geq \text { optimal value of } D P \\
& =\text { optimal value of } D D P .
\end{aligned}
$$

Equality holds if $D D P$ has a rank-1 optimal solution.

We start with a key observation motivated from [22], [27]. To simplify the notation from $D P$, we denote

$x:=\left(\bar{\lambda}_{k}, \underline{\lambda}_{k}, \bar{\mu}_{k}, \underline{\mu}_{k}, k \in[n]\right), r:=\left(\bar{\gamma}_{k}, \underline{\gamma}_{k}, k \in[n]\right)$, and $A(x, r):=I+\sum_{k}\left(\lambda_{k} \Phi_{k}+\mu_{k} \Psi_{k}+\gamma_{k} J_{k}\right)$.

Theorem 3.1: Suppose the dual problem $D P$ is strictly feasible and has a finite optimal solution $\left(x_{*}, r_{*}\right) \geq 0$. If rank $A\left(x_{*}, r_{*}\right)=n-1$ then the duality gap between $P$ and $D P / D D P$ is zero.

Proof: Since $D P$ has a strictly feasible solution, Slater's condition is satisfied and strong duality holds between $D P$ and $D D P$. Let $W_{*} \succeq 0$ be an optimal solution of $D D P$. The complementary slackness condition at the primal-dual optimal point $\left(x_{*}, r_{*}, W_{*}\right)$ of $D P-D D P$ is $\operatorname{tr}\left(A\left(x_{*}, r_{*}\right) W_{*}\right)=0$. Let the positive eigenvalues of $W_{*}$ be $\rho_{i}$ 's and the corresponding eigenvectors be $w_{k}$ 's. Then

$$
\operatorname{tr}\left(A\left(x_{*}, r_{*}\right) W_{*}\right)=\sum_{i=1}^{\operatorname{rank}\left(W_{*}\right)} \rho_{i} w_{i}^{*} A\left(x_{*}, r_{*}\right) w_{i}=0 .
$$

Since $A\left(x_{*}, r_{*}\right) \succeq 0$ and $\rho_{i}>0$ we must have $w_{i}^{*} A\left(x_{*}, r_{*}\right) w_{i}=0$ for all $i$. Thus $w_{i} \in \mathcal{N}\left(A\left(x_{*}, r_{*}\right)\right)$, the null space of $A\left(x_{*}, r_{*}\right)$. Since $w_{i}$ 's span the column space of $W_{*}, \operatorname{rank}\left(W_{*}\right) \leq \operatorname{dim} \mathcal{N}\left(A\left(x_{*}, r_{*}\right)\right)=n-\operatorname{rank} A\left(x_{*}, r_{*}\right)$. Hence if rank $A\left(x_{*}, r_{*}\right)=n-1$ then $W_{*}$ is rank-1 and the proof is complete.

Remark 1: Strict feasibility of $D P$. For strictly feasibility it is sufficient that there is an $(x, r) \geq 0$ such that $A(x, r) \succ 0$, for if such a point has any component that is not strictly positive, say, $\bar{\lambda}_{k} \geq 0, \underline{\lambda}_{k} \geq 0$, we can always replace that component by a strictly positive component $\bar{\lambda}_{k}^{\prime}:=\bar{\lambda}_{k}+\epsilon$, $\underline{\lambda}_{k}^{\prime}:=\underline{\lambda}_{k}+\epsilon$ with $\epsilon>0$, and maintain $A\left(x^{\prime}, r^{\prime}\right) \succ 0$ at this new strictly feasible point.

Remark 2: OPF algorithm when duality gap is zero. In the absence of duality gap, solving the dual problem offers an efficient way to compute an optimal voltage $V_{*}$ for the primal problem. One can solve $D P$ for the $6 n$ variables $\left(x_{*}, r_{*}\right)$, 
construct $A\left(x_{*}, r_{*}\right)$ and verify that it has rank $n-1$, in which case the optimal voltage $V_{*}$ is in its null space. Alternatively, one can solve $D D P$ for an $\frac{1}{2} n(n-1)$-variable optimal $W_{*}$. Since $W_{*}$ is positive semi-definite and rank 1 , it has a unique decomposition $W_{*}=\rho_{*} w_{*} w_{*}^{*}$ where $\rho_{*}>0$ is its positive eigenvalue and $w_{*}$ is the associated eigenvector. Then the optimal voltage is $V_{*}=\sqrt{\rho_{*}} w_{*}$.

We now specialize to radial networks with tree graphs $\mathcal{G}(Y)$ and prove for two cases that rank $A\left(x_{*}, r_{*}\right)$ is indeed $n-1$. We will use the following result from [34, Corollary 3.9] on the minimum rank of matrices with an underlying tree graph.

Lemma 3.2: If an $n \times n$ matrix $H$ is positive semi-definite and the associated graph $\mathcal{G}(H)$ is a connected tree, then rank $H \geq n-1$.

We refer the reader to [34] for its proof. See [35], [36] for surveys on the minimum rank of graphs. The case of Hermitian positive semi-definite matrices are studied in e.g., [37]-[40].

\section{B. Case 1: $\mathcal{G}\left(A\left(x_{*}, r_{*}\right)\right)$ is connected tree}

Lemma 3.2 implies the following characterization of zero duality gap in tree networks.

Theorem 3.3: Suppose Assumption 1 holds. Suppose the dual problem $D P$ is strictly feasible and has a finite optimal solution $\left(x_{*}, r_{*}\right) \geq 0$. If $\left[A\left(x_{*}, r_{*}\right)\right]_{i j} \neq 0$ whenever $Y_{i j} \neq 0$, $i \neq j$, then the duality gap between $P$ and $D P / D D P$ is zero.

Proof: We first show that under Assumption 1, the graph $\mathcal{G}\left(A\left(x_{*}, r_{*}\right)\right)$ consists of possibly more than one tree and follows the same structure as the graph of the underlying network, i.e., we show that, for $i \neq j$, if $Y_{i j}=0$ then $\left[A\left(x_{*}, r_{*}\right)\right]_{i j}=0$. Now, for $i \neq j$

$$
\begin{gathered}
\Phi_{k}(i, j)= \begin{cases}\frac{1}{2} Y_{i j} & \text { if } k=i \\
\frac{1}{2} \bar{Y}_{i j} & \text { if } k=j \\
0 & \text { if } k \neq i, k \neq j\end{cases} \\
\Psi_{k}(i, j)= \begin{cases}\frac{-1}{2 \mathbf{i}} Y_{i j} & \text { if } k=i \\
\frac{1}{2 \mathbf{i}} \bar{Y}_{i j} & \text { if } k=j \\
0 & \text { if } k \neq i, k \neq j,\end{cases}
\end{gathered}
$$

where $\Phi_{k}(i, j)$ and $\Psi_{k}(i, j)$ denote the $(i, j)^{t h}$ entries of these matrices and $\bar{Y}_{i j}$ denotes the complex conjugate of $Y_{i j}$. Hence if $Y_{i j}=0$ then

$$
\begin{aligned}
& {\left[A\left(x_{*}, r_{*}\right)\right]_{i j}=\sum_{k}\left(\lambda_{k} \Phi_{k}(i, j)+\mu_{k} \Psi_{k}(i, j)\right)} \\
& =\frac{1}{2}\left(\lambda_{i} Y_{i j}+\lambda_{j} \bar{Y}_{i j}+\mathbf{i} \mu_{i} Y_{i j}-\mathbf{i} \mu_{j} \bar{Y}_{i j}\right)=0 .
\end{aligned}
$$

This, together with Assumption 1, implies that the (undirected) graph $\mathcal{G}\left(A\left(x_{*}, r_{*}\right)\right)$ has no loops. The condition in the theorem that $\left[A\left(x_{*}, r_{*}\right)\right]_{i j} \neq 0$ whenever $Y_{i j} \neq 0$ then guarantees that $\mathcal{G}\left(A\left(x_{*}, r_{*}\right)\right)$ is a connected tree. Hence by Lemma 3.2 rank $A\left(x_{*}, r_{*}\right)=n-1$, whenever $W_{*} \neq 0$ (i.e., $V_{*} \neq 0$ and we have a nontrivial solution) and the claim follows from Theorem 3.1 .
Without the condition in Theorem 3.3, $A\left(x_{*}, r_{*}\right)$ may have a zero off-diagonal entry where $Y$ has a nonzero entry and $\mathcal{G}\left(A\left(x_{*}, r_{*}\right)\right)$ may consist of a collection of disjoint trees. In this case the rank of $A\left(x_{*}, r_{*}\right)$ may be strictly less than $n-1$ and we cannot rely on Theorem 3.1 to prove zero duality gap.

\section{Case 2: Load can be over-satisfied}

From (4) the graph $\mathcal{G}\left(A\left(x_{*}, r_{*}\right)\right)$ is indeed a connected tree if, for any buses $i$ and $j$ that are connected (i.e., $Y_{i j} \neq 0$ ), all of $\lambda_{i}, \mu_{i}, \lambda_{j}, \mu_{j}$ are nonnegative and at least one of them is strictly positive. This motivates the case where the loads can be over-satisfied, i.e., the real and imaginary powers supplied to a node can be greater than the real and imaginary powers demanded by them respectively. This corresponds to the case where the real and reactive power constraints in problem $P$ do not have lower bounds. In this case the Lagrange multipliers $\lambda$ and $\mu$ are indeed nonnegative. Note that the problem still remains non-convex as the matrices $\Phi_{k}$ and $\Psi_{k}$ are generally indefinite. Hence we consider the following:

\section{Modified Primal Problem $(m P)$ :}

$$
\begin{array}{cl}
\underset{V}{\operatorname{minimize}} & V^{*} V \\
\text { subject to } & V^{*} \Phi_{k} V \leq \bar{P}_{k}, \quad k \in[n] \\
& V^{*} \Psi_{k} V \leq \bar{Q}_{k}, \quad k \in[n] \\
& \underline{W}_{k} \leq V^{*} J_{k} V \leq \bar{W}_{k}, \quad k \in[n] .
\end{array}
$$

Let $\bar{\lambda}=\left(\bar{\lambda}_{k}, k \in[n]\right)$ be the Lagrange multipliers corresponding to the upper inequalities for the real power and $\bar{\mu}=\left(\bar{\mu}_{k}, k \in[n]\right)$ be those for the reactive power. We consider both-sided inequalities on the voltages and hence $r=\left(\bar{\gamma}_{k}, \underline{\gamma}_{k}, k \in[n]\right)$ remains the same. The definition of $A$ naturally carries over: $A(\bar{\lambda}, \bar{\mu}, r):=I+\sum_{k}\left(\bar{\lambda}_{k} \Phi_{k}+\right.$ $\left.\bar{\mu}_{k} \Psi_{k}+\gamma_{k} J_{k}\right)$ where $\gamma_{k}:=\bar{\gamma}_{k}-\underline{\gamma}_{k}$ as before. Let $\bar{P}:=\left(\bar{P}_{k}, k \in[n]\right)$ be the upper bounds on the real power, $\bar{Q}:=\left(\bar{Q}_{k}, k \in[n]\right)$ be those on the reactive power, and $d:=\left(\bar{W}_{k},-\underline{W}_{k}, k \in[n]\right)$ be the upper and lower bounds on the voltages. Consider the following pair of problems:

Dual of $m P(m D P)$ :

$$
\begin{array}{ll}
\underset{\bar{\lambda}, \bar{\mu}, r \geq 0}{\operatorname{maximize}} & -\bar{\lambda}^{T} \bar{P}-\bar{\mu}^{T} \bar{Q}-d^{T} r \\
\text { subject to } & A(\bar{\lambda}, \bar{\mu}, r) \succeq 0, \quad \bar{\lambda} \geq 0, \bar{\mu} \geq 0 .
\end{array}
$$

Dual of $m D P(m D D P)$ :

$$
\begin{array}{cl}
\underset{W, \alpha, \beta}{\operatorname{minimize}} & \operatorname{tr}(W) \\
\text { subject to } & \operatorname{tr}\left(\Phi_{k} W\right)+\alpha_{k}=\bar{P}_{k}, \quad k \in[n] \\
& \operatorname{tr}\left(\Psi_{k} W\right)+\beta_{k}=\bar{Q}_{k}, \quad k \in[n] \\
& \underline{W}_{k} \leq \operatorname{tr}\left(J_{k} W\right) \leq \bar{W}_{k}, \quad k \in[n] \\
& W \succeq 0, \quad \alpha \geq 0, \quad \beta \geq 0,
\end{array}
$$

where $\alpha, \beta$ are the Lagrange multipliers corresponding to the constraints $\bar{\lambda}, \bar{\mu} \geq 0$. It is clear that the modified primal problem $m P$ is equivalent to the problem $m D D P$ with the 
additional constraint that rank $W=1$, and that strong duality holds between $m D P$ and $m D D P$ provided that Slater's condition is satisfied. Our main result is

Theorem 3.4: Suppose Assumption 1 holds. Suppose the dual problem $m D P$ is strictly feasible and has a finite optimal solution $\left(\bar{\lambda}_{*}, \bar{\mu}_{*}, r_{*}\right) \geq 0$. Then the duality gap between $m P$ and $m D P / m D D P$ is zero.

Proof: Since $m D P$ is strictly feasible, Slater's condition is satisfied and strong duality holds between $m D P$ and $m D D P$. Hence, as for the unmodified problems, we have

$$
\text { optimal value of } \begin{aligned}
m P & \geq \text { optimal value of } m D P \\
& =\text { optimal value of } m D D P .
\end{aligned}
$$

Theorem 3.1 implies that the duality gap between $m P$ and its dual $m D P$ is zero if the matrix $A\left(\bar{\lambda}_{*}, \bar{\mu}_{*}, r_{*}\right)$ has rank $n-1$. The KKT conditions for the pair of problems $m D P$ and $m D D P$ consist of: primal feasibility (5), dual feasibility (6)-(9), the complementary slackness

$$
\operatorname{tr}\left(A\left(\bar{\lambda}_{*}, \bar{\mu}_{*}, r_{*}\right) W_{*}\right)=0, \quad \alpha_{*}^{T} \bar{\lambda}_{*}=0, \quad \beta_{*}^{T} \bar{\mu}_{*}=0
$$

and the gradient condition for primal optimality

$$
\begin{gathered}
\left(\bar{\gamma}_{*}\right)_{k}\left(\operatorname{tr}\left(J_{k} W_{*}\right)-\bar{W}_{k}\right)=0, \quad k \in[n] \\
\left(\underline{\gamma}_{*}\right)_{k}\left(\operatorname{tr}\left(J_{k} W_{*}\right)-\underline{W}_{k}\right)=0, \quad k \in[n] .
\end{gathered}
$$

Any set of variables $\left(\bar{\lambda}_{*}, \bar{\mu}_{*}, r_{*}, W_{*}, \alpha_{*}, \beta_{*}\right)$ that satisfies the KKT conditions is optimal for the primal-dual pair $m D P-$ $m D D P$. We now construct such a point with rank $W_{*}=1$.

By Lemma 3.2 and Theorem 3.1, to prove that $W_{*}$ is rank-1, it suffices to show that the graph $\mathcal{G}\left(A\left(\bar{\lambda}_{*}, \bar{\mu}_{*}, r_{*}\right)\right)$ is a connected tree. This requires that $\left[A\left(\bar{\lambda}_{*}, \bar{\mu}_{*}, r_{*}\right)\right]_{i j} \neq 0$ wherever $Y_{i j} \neq 0$, which however may not be true for

$$
\begin{array}{r}
\operatorname{Re}\left\{A\left(\bar{\lambda}_{*}, \bar{\mu}_{*}, r_{*}\right)\right\}_{i j} \\
=-\frac{1}{2}\left[g_{i j}\left(\bar{\lambda}_{i}+\bar{\lambda}_{j}\right)+b_{i j}\left(\bar{\mu}_{i}+\bar{\mu}_{j}\right)\right] .
\end{array}
$$

Since $\bar{\lambda}_{k}, \bar{\mu}_{k}$ are only nonnegative but not necessarily positive, it is possible that $\left[A\left(\bar{\lambda}_{*}, \bar{\mu}_{*}, r_{*}\right)\right]_{i j}=0$ but $Y_{i j} \neq 0$ for some link $(i, j)$, i.e., $\mathcal{G}\left(A\left(\bar{\lambda}_{*}, \bar{\mu}_{*}, r_{*}\right)\right)$ may not be connected even when $\mathcal{G}(Y)$ is. To deal with this problem, we consider a sequence of problems, each of which has $(\bar{\lambda}, \bar{\mu})>0$ and therefore has a rank-1 solution (by Lemma 3.2 and Theorem 3.1), and prove that the sequence converges to the pair $m D P-m D D P$.

Specifically consider the $\epsilon$-shifted problem $m D P^{\epsilon}$ where we replace the constraint $(\bar{\lambda}, \bar{\mu}) \geq 0$ by $(\bar{\lambda}, \bar{\mu}) \geq \epsilon \mathbf{1}$ where $\epsilon>0$ and $\mathbf{1}$ is a vector of all 1's of appropriate size. This changes $m D D P$ to a $\epsilon$-shifted problem $m D D P^{\epsilon}$ whose objective function becomes $\operatorname{tr} W-\epsilon \mathbf{1}^{T}(\alpha+\beta)$ but the constraints remain the same as those of $m D D P$. The KKT conditions for $m D P^{\epsilon}-m D D P^{\epsilon}$ differ from those of $m D P-m D D P$ only in part of the primal feasibility condition in (5) and the corresponding complementary slackness condition in (10), as follows:

$$
\begin{aligned}
\bar{\lambda}_{*}(\epsilon) \geq \epsilon \mathbf{1}, & \bar{\mu}_{*}(\epsilon) \geq \epsilon \mathbf{1} \\
\alpha_{*}(\epsilon)^{T}\left(\bar{\lambda}_{*}(\epsilon)-\epsilon \mathbf{1}\right)=0, \quad & \beta_{*}^{T}(\epsilon)\left(\bar{\mu}_{*}(\epsilon)-\epsilon \mathbf{1}\right)=0 .
\end{aligned}
$$

All other conditions remain the same. Moreover we can choose small enough $\epsilon_{0}>0$ such that $m D P^{\epsilon_{0}}$ remains strictly feasible and hence strong duality holds between $m D P^{\epsilon_{0}}$ and $m D D P^{\epsilon_{0}}$. Also, since the feasible set of $m D P^{\epsilon_{0}}$ is a subset of $m D P$, there exists a finite optimal $\left(\bar{\lambda}_{*}\left(\epsilon_{0}\right), \bar{\mu}_{*}\left(\epsilon_{0}\right), r_{*}\left(\epsilon_{0}\right)\right)$ that solves $m D P_{0}^{\epsilon}$ by continuity of the objective function.

For any $0<\epsilon<\epsilon_{0}$, the linearity of $m D P$ implies that there is an optimal solution $\left(\bar{\lambda}_{*}(\epsilon), \bar{\mu}_{*}(\epsilon), r_{*}(\epsilon)\right)$ that lies on the line segment between the given $\left(\bar{\lambda}_{*}, \bar{\mu}_{*}, r_{*}\right)$ and $\left(\bar{\lambda}_{*}\left(\epsilon_{0}\right), \bar{\mu}_{*}\left(\epsilon_{0}\right), r_{*}\left(\epsilon_{0}\right)\right)$. Hence these optimal points $\left(\bar{\lambda}_{*}(\epsilon), \bar{\mu}_{*}(\epsilon), r_{*}(\epsilon)\right)$ for all $\epsilon \in\left(0, \epsilon_{0}\right)$ live in a compact set independent of $\epsilon$. Since the constraints (6)-(9) of $D D P$ are the same as those of $m D D P^{\epsilon}$ and are independent of $\epsilon$, the optimal solutions $\left(W_{*}(\epsilon), \alpha_{*}(\epsilon), \beta_{*}(\epsilon)\right)$ for every $\epsilon$ also live in a fixed compact set. Hence as we take $\epsilon \rightarrow 0$, there is a subsequence of the set of primal-dual optimal points $\left(\bar{\lambda}_{*}(\epsilon), \bar{\mu}_{*}(\epsilon), r_{*}(\epsilon), W_{*}(\epsilon), \alpha_{*}(\epsilon), \beta_{*}(\epsilon)\right)$ that converges. Let the limit be $\left(\bar{\lambda}_{*}, \bar{\mu}_{*}, r_{*}, W_{*}^{\prime}, \alpha_{*}^{\prime}, \beta_{*}^{\prime}\right)$. Clearly, this point satisfies the KKT conditions, (5), (6)-(9), (10), (11)-(12), and hence is primal-dual optimal for $m D P-m D D P$. We are left to show that $W_{*}$ is rank 1 .

For each such $\epsilon \in\left(0, \epsilon_{0}\right)$, (13) and Assumption 1 imply that $\mathcal{G}\left(A\left(\bar{\lambda}_{*}(\epsilon), \bar{\mu}_{*}(\epsilon), r_{*}(\epsilon)\right)\right)$ is a connected tree. Hence $A\left(\bar{\lambda}_{*}(\epsilon), \bar{\mu}_{*}(\epsilon), r_{*}(\epsilon)\right)$ has rank $n-1$ and $W_{*}(\epsilon)$ has rank $1\left(W_{*}(\epsilon) \neq 0\right.$ because the voltage constraints make the diagonal elements nonzero ). Since the set of positive semidefinite matrices with rank $\leq 1$ is closed [41], the limit $W_{*}^{\prime}$ of the convergent subsequence can have at most rank 1. By construction $W_{*}^{\prime}=\left(V_{*}^{\prime}\right)\left(V_{*}^{\prime}\right)^{*}$ and $V_{*}^{\prime} \neq 0$ is not feasible, $W_{*}^{\prime}$ must have rank 1 . This completes the proof.

\section{Extension: with storage}

Assume that every node $k$ in the network has some storage element (e.g., a battery) with finite energy capacity $B_{k}$. Consider discrete time $t=1, \ldots, T$, where $b_{k}(t)$ denotes the state of charge of the storage at node $k$ and time $t$. The ramp rate of the storage is constrained such that

$$
\underline{D_{k}} \leq b_{k}(t+1)-b_{k}(t) \leq \overline{D_{k}}, \quad t \in[1, T-1] .
$$

Given an initial state of the storage $0 \leq \mathfrak{b}_{k}^{0} \leq B_{k}$, for $k \in$ $[n]$, the OPF with storage problem becomes:

Primal Problem with Storage $(S P)$ :

$$
\begin{array}{cl}
\underset{\substack{V(t), b_{k}(t) \text { for } k \in[n] \\
\text { subject to }}}{\operatorname{minimize}} & \sum_{t=1}^{T} V(t)^{*} V(t) \\
& \underline{P}_{k}(t) \leq V(t)^{*} \Phi_{k} V(t) \leq \bar{P}_{k}(t) \\
& \underline{Q}_{k}(t) \leq V(t)^{*} \Psi_{k} V(t) \leq \bar{Q}_{k}(t) \\
& \underline{W}_{k}(t) \leq V(t)^{*} J_{k} V(t) \leq \bar{W}_{k}(t) \\
& 0 \leq b_{k}(t) \leq B_{k} \\
& \underline{D_{k}} \leq b_{k}(t+1)-b_{k}(t) \leq \overline{D_{k}} \\
& \text { for } t \in[1, T-1] \\
& b_{k}(1)=\mathfrak{b}_{k}^{0}
\end{array}
$$


where $k \in[n]$ and $t=1, \ldots, T$, unless otherwise indicated.

As in [42], the addition of storage charge/discharge dynamics yields a dual problem with storage $(S D P)$ that has an LMI condition of the form $A(x, r)(t)$ for each $t=1, \ldots, T$. The structure of this matrix at each $t$ remains the same as in the original $D P$ (or $m D P$ for Case 2: Load is over-satisfied) so, the results described in sections III-A-III-C carry over to the $S P$ case.

\section{CONCLUSiON}

This paper examined power network optimization over radial (tree) networks, which is the topology commonly found in distribution systems. As in previous works, we show that the OPF problem can be reformulated as a rank constrained (i.e. non-convex) semi-definite program, with a sufficient condition regarding when the rank constraint is satisfied. We introduce a complex formulation that simplifies this sufficient condition and then show that in a radial network this condition is always met provided we allow oversatisfaction of load. In other words, we prove that if the loads are over-satisfied, then the duality gap for OPF over a tree network is always zero. The proof technique relies on the fact that if the underlying graph of an $n \times n$ Hermitian positive semi-definite matrix is a tree, then the matrix has rank at least $n-1$. Our results extend to the case where simple distributed storage dynamics are added to the problem.

For future work, we will investigate the conditions for zero duality gap without the load over-satisfaction assumptions and study the effectiveness of the proposed algorithm using practical distribution (radial) test circuits. Further extensions will include analysis of the more general OPF problem and more extensive study regarding the underlying system properties that yield zero duality gap solutions for most practical circuit models.

\section{ACKNOWLEDGEMENTS}

A special thank you to Christopher Clarke of Southern California Edison for providing insightful comments and a number of interesting discussions.

\section{REFERENCES}

[1] J. Carpentier, "Contribution to the economic dispatch problem," Bulletin de la Societe Francoise des Electriciens, vol. 3, no. 8, pp. 431447, 1962, in French.

[2] J. A. Momoh, Electric Power System Applications of Optimization, ser. Power Engineering, H. L. Willis, Ed. Markel Dekker Inc.: New York, USA, 2001.

[3] W. W. Hogan, "Contract networks for electric power transmission," J. of Regulatory Economics, vol. 4, no. 3, pp. 211-42, 1992.

[4] H. P. Chao and S. Peck, "A market mechanism for electric power transmission," J. of Regulatory Economics, vol. 10, pp. 25-59, 1996.

[5] B. Stott and O. Alsaç, "Fast decoupled load flow," IEEE Trans. on Power Apparatus and Systems, vol. PAS-93, no. 3, pp. 859-869, 1974.

[6] O. Alsaç, J. Bright, M. Prais, and B. Stott, "Further developments in LP-based optimal power flow," IEEE Trans. on Power Systems, vol. 5, no. 3, pp. 697-711, 1990.

[7] K. Purchala, L. Meeus, D. Van Dommelen, and R. Belmans, "Usefulness of DC power flow for active power flow analysis," in Proc. of IEEE PES General Meeting. IEEE, 2005, pp. 2457-2462.

[8] B. Stott, J. Jardim, and O. Alsaç, "DC Power Flow Revisited," IEEE Trans. on Power Systems, vol. 24, no. 3, pp. 1290-1300, Aug. 2009.
[9] M. Huneault and F. D. Galiana, "A survey of the optimal power flow literature," IEEE Trans. on Power Systems, vol. 6, no. 2, pp. 762-770, 1991.

[10] J. A. Momoh, M. E. El-Hawary, and R. Adapa, "A review of selected optimal power flow literature to 1993. Part I: Nonlinear and quadratic programming approaches," IEEE Trans. on Power Systems, vol. 14 no. 1, pp. 96-104, 1999.

[11] _ _A review of selected optimal power flow literature to 1993. Part II: Newton, linear programming and interior point methods," IEEE Trans. on Power Systems, vol. 14, no. 1, pp. 105 - 111, 1999.

[12] K. S. Pandya and S. K. Joshi, "A survey of optimal power flow methods," J. of Theoretical and Applied Information Technology, vol. 4, no. 5, pp. 450-458, 2008.

[13] R. Jabr, "Radial Distribution Load Flow Using Conic Programming," IEEE Trans. on Power Systems, vol. 21, no. 3, pp. 1458-1459, Aug. 2006.

[14] R. A. Jabr, "A Conic Quadratic Format for the Load Flow Equations of Meshed Networks," IEEE Trans. on Power Systems, vol. 22, no. 4 pp. 2285-2286, Nov. 2007.

[15] R. Jabr, "Optimal Power Flow Using an Extended Conic Quadratic Formulation," IEEE Trans. on Power Systems, vol. 23, no. 3, pp. 10001008, Aug. 2008.

[16] M. E. Baran and F. F. Wu, "Optimal Capacitor Placement on radial distribution systems," IEEE Trans. Power Delivery, vol. 4, no. 1, pp. 725-734, 1989

[17] _ , "Optimal Sizing of Capacitors Placed on A Radial Distribution System," IEEE Trans. Power Delivery, vol. 4, no. 1, pp. 735-743, 1989.

[18] M. Farivar, C. R. Clarke, S. H. Low, and K. M. Chandy, "Inverter VAR control for distribution systems with renewables," 2011, int'l Conf. on Smart Grid Communications (SmartGridComm), to appear

[19] X. Bai, H. Wei, K. Fujisawa, and Y. Wang, "Semidefinite programming for optimal power flow problems," Int'l J. of Electrical Power \& Energy Systems, vol. 30, no. 6-7, pp. 383-392, 2008.

[20] X. Bai and H. Wei, "Semi-definite programming-based method for security-constrained unit commitment with operational and optimal power flow constraints," Generation, Transmission \& Distribution, IET, vol. 3, no. 2, pp. 182-197, 2009

[21] J. Lavaei, "Zero duality gap for classical OPF problem convexifies fundamental nonlinear power problems," in Proc.of the American Control Conf., 2011.

[22] J. Lavaei and S. Low, "Zero duality gap in optimal power flow problem," IEEE Trans. on Power Systems, To Appear, 2011.

[23] Y. Ding, "On efficient semidefinite relaxations for quadratically constrained quadratic programming," Mathematics, Univ. of Waterloo, 2007.

[24] S. Kim and M. Kojima, "Exact solutions of some nonconvex quadratic optimization problems via SDP and SOCP relaxations," Computational Optimization and Applications, vol. 26, no. 2, pp. 143-154, 2003.

[25] S. Zhang, "Quadratic maximization and semidefinite relaxation," Mathematical Programming, vol. 87, no. 3, pp. 453-465, 2000.

[26] Z. Luo, W. Ma, A. So, Y. Ye, and S. Zhang, "Semidefinite relaxation of quadratic optimization problems," Signal Processing Magazine, IEEE, vol. 27, no. 3, pp. $20-34$, May 2010.

[27] H. Wolkowicz, R. Saigal, and L. Vandenberghe, Handbook of Semidefinite Programming: Theory, Algorithms, and Applications. Springer Netherlands, 2000, vol. 27.

[28] M. Goemans and D. Williamson, "Improved approximation algorithms for maximum cut and satisfiability problems using semidefinite programming," Journal of the ACM (JACM), vol. 42, no. 6, pp. 1115 1145,1995

[29] M. McCoy and J. Tropp, "Two proposals for robust pca using semidefinite programming," Arxiv preprint arXiv:1012.1086, 2010.

[30] S. Boyd and L. Vandenberghe, Convex Optimization. Cambridge Univ. Press, 2004.

[31] K. P. Schneider, Y. Chen, D. P. Chassin, R. Pratt, D. Engel, and S. Thompson, "Modern Grid Initiative: Distribution Taxonomy Final Report Table of Contents," US Department of Energy, Tech. Rep., 2008.

[32] K. Turitsyn, P. Sŭlc, S. Backhaus, and M. Chertkov, "Options for control of reactive power by distributed photovoltaic generators," Proc. of the IEEE, vol. 99, no. 6, pp. 1063 -1073, June 2011.

[33] K. Schneider, J. Fuller, F. Tuffner, and S. R., "Evaluation of conservation voltage reduction (CVR) on a national level," Pacific Northwest National Laboratory, Tech. Rep., 2010. 
[34] C. Johnson, A. Leal Duarte, C. Saiago, B. Sutton, and A. Witt, "On the relative position of multiple eigenvalues in the spectrum of an Hermitian matrix with a given graph," Linear Algebra and its Applications, vol. 363, pp. 147-159, 2003.

[35] S. Fallat and L. Hogben, "Variants on the minimum rank problem: A survey II," Arxiv preprint arXiv:1102.5142, 2011.

[36] - "The minimum rank of symmetric matrices described by a graph: A survey," Linear Algebra and its Applications, vol. 426, no. 2-3, pp. 558-582, 2007.

[37] M. Booth, P. Hackney, B. Harris, C. Johnson, M. Lay, L. Mitchell, S. Narayan, A. Pascoe, K. Steinmetz, B. Sutton, et al., "On the minimum rank among positive semidefinite matrices with a given graph," SIAM J. on Matrix Analysis and Applications, vol. 30, no. 2, pp. 731-740, 2008.
[38] P. Hackney, B. Harris, M. Lay, L. Mitchell, S. Narayan, and A. Pascoe, "Linearly independent vertices and minimum semidefinite rank," Linear Algebra and Its Applications, vol. 431, no. 8, pp. 1105-1115, 2009.

[39] Y. Jiang, K. Kudryavtseva, J. Marinova, and S. Narayan, "Minimum Positive Semi-Definite Rank of a Graph," Central Michigan Univ., Tech. Rep., 2005.

[40] Y. Jiang, L. Mitchell, and S. Narayan, "Unitary matrix digraphs and minimum semidefinite rank," Linear Algebra and its Applications, vol. 428, no. 7, pp. 1685-1695, 2008.

[41] R. Horn and C. Johnson, Matrix Analysis. Cambridge Univ. Press, 2005.

[42] D. Gayme and U. Topcu, "Optimal power flow with distributed energy storage dynamics," in Proc. of the American Control Conf., 2011. 